\title{
MicroRNA-122 in heart failure with reduced ejection fraction: Epiphenomenon or causal?
}

\author{
Aldo Bonaventura ${ }^{\mathrm{a}, \mathrm{b}}$, Alessandra Vecchié ${ }^{\mathrm{a}, \mathrm{b}}$, Sarah Costantino $^{\mathrm{c}}$, Francesco Paneni ${ }^{\mathrm{c}, \mathrm{d}, \mathrm{e}, *}$ \\ ${ }^{a}$ Virginia Commonwealth University, Pauley Heart Center, Division of Cardiology, Department of Internal Medicine, Richmond, VA, United States \\ ${ }^{\mathrm{b}}$ First Clinic of Internal Medicine, Department of Internal Medicine, University of Genoa, Genoa, Italy \\ c Center for Molecular Cardiology, Schlieren Campus, University of Zurich, 8952 Schlieren, Switzerland \\ d Department of Cardiology, University Hospital Zurich, 8091 Zurich, Switzerland \\ e Department of Research and Education, University Hospital Zurich, 8001 Zurich, Switzerland
}

Heart failure (HF) is nowadays one of the most common diagnoses for hospital admission and this trend is expected to persist due to the ageing of the world population [1]. Although advances in HF therapy [2], the prognosis of these patients remains poor and further efforts are required to enable an early diagnosis, risk stratification and personalized therapies. Albeit our genetic profile strongly contributes to determine HF risk, growing evidence indicates that epi-genetic changes including DNA methylation, histone modifications and non-coding RNAs (ncRNAs) - may act as fundamental biological layers regulating gene expression and cardiovascular phenotype over the lifetime. Among the ncRNAs family, microRNAs (miRNAs) have been extensively investigated in the setting of cardiovascular disease and HF. MiRNAs are 22-nucleotide long structures involved in different cellular processes through the blockade of messenger RNA translation by binding at the complementary 3 '-untranslated region and modulating gene expression at the post-transcriptional level [3]. A wealth of miRNAs were reported to have a role in cardiac remodeling, hypertrophy, hypoxia, and apoptosis eventually fostering HF development [4-6]. Whether miRNAs can be employed as reliable HF biomarkers remains to be fully elucidated.

In this issue of the Journal, Stojkovic et al. investigated the role of the liver-specific miRNA-122 as a marker for prognosis and risk stratification in a case-control cohort of 40 patients with HF with reduced ejection fraction (HFrEF) who died during the follow-up (cases) and 36 survivors (controls) [7]. Among 84 miRNAs evaluated in the screening phase, only 3 miRNAs were differentially expressed among the 2 groups: miRNA-122, -423 , and -126 . The levels of these miRNAs were then further assessed in a validation cohort of 234 patients with $\mathrm{HFrEF}$ at the time of outpatient clinic admission. Seventy-six patients died during follow-up (median 3.2 years), of whom 52 for a cardiovascular cause. Levels of miRNA-122 and -423 were found higher among non-survivor patients and both displayed positive correlations with $\mathrm{N}$ -

\footnotetext{
DOI of original article: https://doi.org/10.1016/j.ijcard.2019.11.090.

* Corresponding author at: Center for Molecular Cardiology, Schlieren Campus, University of Zurich, 8952 Schlieren, Switzerland.

E-mail address: francesco.paneni@uzh.ch (F. Paneni).
}

terminal (NT)-pro hormone B-type natriuretic peptide (NT-proBNP) levels. Furthermore, miRNA-122 and -423 were shown to be independent predictors of all-cause mortality, even after adjustment for relevant confounders such as sex, age, left ventricular ejection fraction, NT-proBNP, diabetes, glomerular filtration rate, NYHA class, right ventricular dysfunction, and previous MI. Finally, the authors found different cut-off levels for the two miRNAs (12 and 3694 copies/ $\mu \mathrm{L}$ plasma, respectively), which were able to identify patients at risk of all-cause mortality and cardiovascular death. Moreover, an additive effect in predicting mortality was found when both miRNAs were above the respective cut-offs. The interesting aspect of the research, therefore, relies on the fact that when miRNA-122 and -423 were separately included in the prediction model, only miRNA-122 significantly improved the prediction for all-cause and cardiovascular mortality on top of classical risk factors and NT-proBNP [7].

The paper by Stojkovic et al. provides new evidence on the role of epigenetic biomarkers, namely miRNAs, in HFrEF patients. While previous work has extensively investigated the role of miRNAs in HF diagnosis and stratification, only few studies focused on their ability to predict prognosis $[6,8,9]$. The latter aspect is of paramount importance as an increasing number of patients will suffer from HFrEF in the decades to come, and reliable circulating biomarkers are in high demand.

MiRNA-122 is the most abundant ncRNA in the liver, where it regulates genes involved in cholesterol and fatty acid metabolism. Here, miRNA-122 was found to independently predict all-cause mortality, even after adjustment for right ventricular dysfunction, cholinesterase and gamma-GT, suggesting that factors other than fluid congestion and liver injury may contribute to explain the association of miR-122 with mortality. In the present study the authors postulated that high levels of miR-122 in HFrEF likely reflect liver damage due to chronic congestion. However, liver-derived miR-122 may also exert its biological effects on the myocardium. Indeed, recent work has shown that inhibition of mir-122 prevents cardiomyocyte apoptosis in an experimental model of atrial fibrillation [10]. In other words, upregulation of miR-122 driven by liver injury may lead to detrimental effects on the heart, thus feeding a noxious vicious cycle. Moreover, myocardial expression of miR-122 may also contribute to the pathophysiology of HF. Although these data suggest a central role of this miR in HF, in the 
study by Stojkovic et al. miRNA-122 was not found to be an independent predictor of cardiovascular mortality. However, miRNA-122 levels showed a significant correlation with NT-proBNP and significantly improved the prognostic model with respect to all-cause and cardiovascular mortality [7].

It is then clear that miRNA-122 might be considered when evaluating patients with $\mathrm{HFrEF}$ in order identify those patients with congestion, liver injury, and at high risk for death. There remain, however, few issues to be addressed before considering mir-122 as a potential biomarker to be employed in the clinical setting. Most of studies conducted so far, including that by Stojkovic et al., did not use a large miRNA panel for screening, thus leading to a potential selection bias. Additionally, the diagnostic and prognostic power of miRNAs should be always compared to currently available, gold standard parameters, such as NT-proBNP, in order to highlight their incremental value in predicting HF-related events. Finally, the lack of consistency in the available studies is worth being discussed as many factors might contribute to these results [3]. For example, different anticoagulants contained in the tubes for blood collection as well as the biological material used for the analysis (plasma, serum, or whole blood) may affect miRNA expression. Unfortunately, we still lack standardized approaches for the assessment of circulating miRNAs, this also represents a major confounder when comparing different studies. Overall, evidence discussed so far does not allow to fully understand whether miR-122 plays any active role in $\mathrm{HFrEF}$, or whether its upregulation reflects liver damage as the result of fluid congestion.

In conclusion, the paper by Stojkovic et al. provides novel insights on miR-122 as an early biomarker of liver injury and mortality in patients with HFrEF. Further studies in larger cohorts are urgently needed to replicate these results.

\section{Declaration of competing interest}

None.

\section{References}

[1] E. Braunwald, The war against heart failure: the Lancet lecture, Lancet 385 (2015) 812-824.

[2] A. Bonaventura, G.F. Wohlford, A. Vecchie, B.W. Van Tassel, A. Abbate, In-hospital initiation of sacubitril/valsartan: a new paradigm for acute decompensated heart failure? J. Cardiovasc. Pharmacol. 74 (2019) 1-3.

[3] E.L. Vegter, P. van der Meer, L.J. de Windt, Y.M. Pinto, A.A. Voors, MicroRNAs in heart failure: from biomarker to target for therapy, Eur. J. Heart Fail. 18 (2016) 457-468.

[4] A.J. Tijsen, Y.M. Pinto, E.E. Creemers, Non-cardiomyocyte microRNAs in heart failure Cardiovasc. Res. 93 (2012) 573-582.

[5] S. Costantino, F. Paneni, T.F. Luscher, F. Cosentino, MicroRNA profiling unveils hyperglycaemic memory in the diabetic heart, Eur. Heart J. 37 (2016) 572-576.

[6] S. Costantino, A. Akhmedov, G. Melina, S.A. Mohammed, A. Othman, S. Ambrosini, et al., Obesity-induced activation of JunD promotes myocardial lipid accumulation and metabolic cardiomyopathy, Eur. Heart J. 40 (2019) 997-1008.

[7] S. Stojkovic, L. Koller, P. Sulzgruber, M. Hulsmann, K. Huber, M. Mayr, et al., Liverspecific microRNA-122 as prognostic biomarker in patients with chronic systolic heart failure, Int. J. Cardiol. (2019) https://doi.org/10.1016/j.ijcard.2019.11.090.

[8] S. Masson, S. Batkai, J. Beermann, C. Bar, A. Pfanne, S. Thum, et al., Circulating microRNA-132 levels improve risk prediction for heart failure hospitalization in patients with chronic heart failure, Eur. J. Heart Fail. 20 (2018) 78-85.

[9] E.S. Ovchinnikova, D. Schmitter, E.L. Vegter, J.M. Ter Maaten, M.A. Valente, L.C. Liu, et al., Signature of circulating microRNAs in patients with acute heart failure, Eur. J. Heart Fail. 18 (2016) 414-423.

[10] S. Xiong, G. Salazar, A. San Martin, M. Ahmad, N. Patrushev, L. Hilenski, et al., PGC-1 alpha serine 570 phosphorylation and GCN5-mediated acetylation by angiotensin II drive catalase down-regulation and vascular hypertrophy, J. Biol. Chem. 285 (2010) 2474-2487. 